Ronald BeLlefontAINE ${ }^{1}$

Abderrahim FERRADOUS ${ }^{2}$

Mohamed ALIFRIQUI ${ }^{3}$

Olivier MONTEUUIS ${ }^{4}$

${ }^{1}$ Cirad

Upr Génétique forestière

F-34398 Montpellier

France

${ }^{2}$ Centre Régional de Recherche Forestière 4000 Marrakech Maroc

${ }^{3}$ Université Caddi Ayyad Faculté des Sciences Semlalia Laboratoire d'écologie et d'environnement 4000 Marrakech

Maroc

${ }^{4}$ Cirad

Umr Dap 1098

F-34398 Montpellier

France

\section{Multiplication végétative de l'arganier, Argania spinosa, au Maroc : le projet John GoELET}

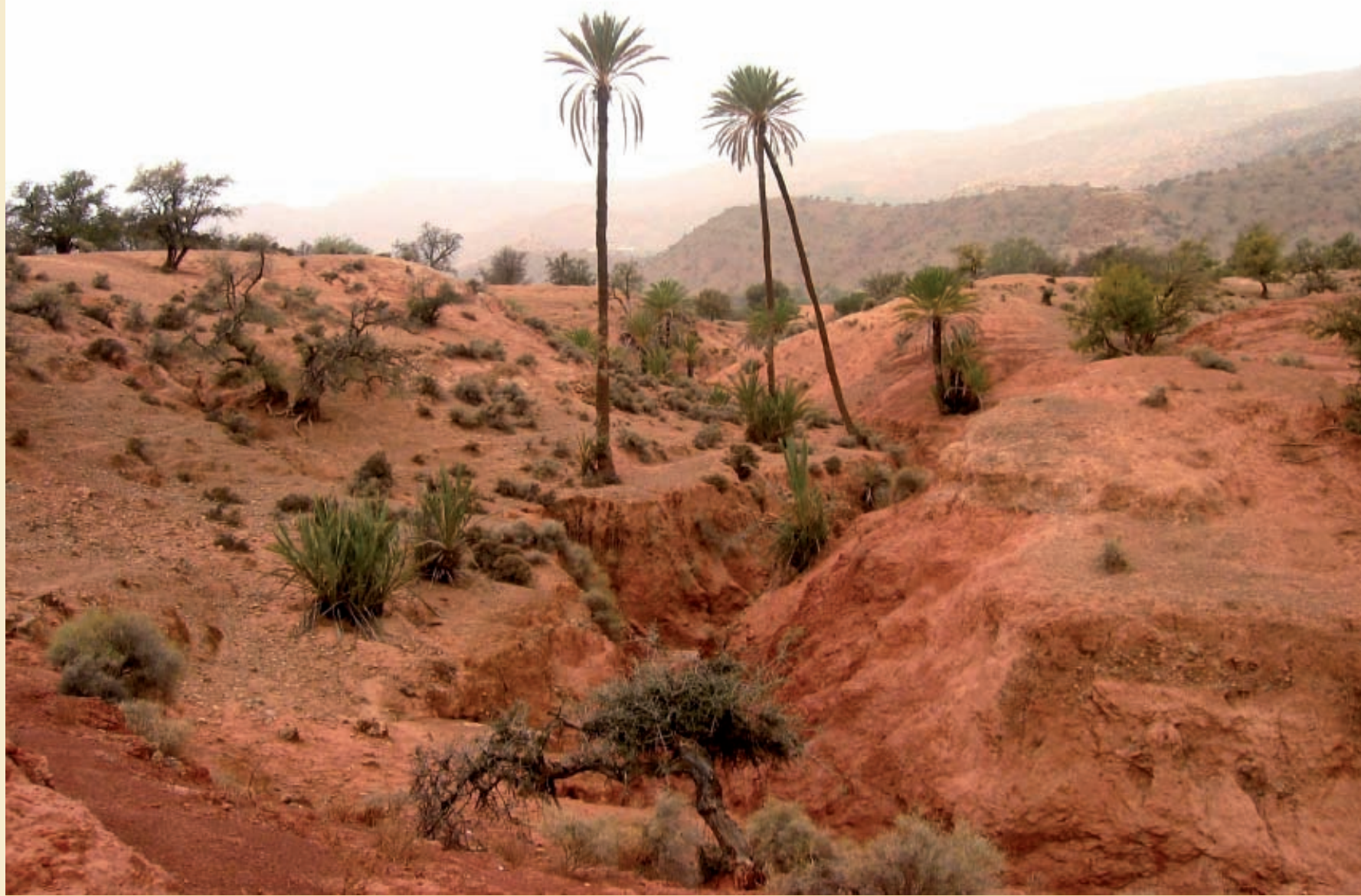

Photo 1.

Arganeraie de montagne dégradée dans le secteur d'Argana. Photo O. Monteuuis. 


\section{MULTIPLICATION VÉGÉTATIVE DE L'ARGANIER, ARGANIA SPINOSA, AU MAROC : LE PROJET JOHN GOELET}

L'arganier est un arbre à usages multiples de grand intérêt socio-économique pour le Sud-Ouest marocain. Il est utilisé pour l'alimentation des hommes et du bétail, en médecine, en cosmétique, tout en permettant de lutter contre la désertification et l'érosion en produisant du bois à diverses finalités. Les peuplements naturels, en constante diminution depuis le XIX siècle, sont menacés par diverses pressions anthropiques, de plus en plus fortes, réduisant les capacités de régénération naturelle de l'espèce. Dans ce contexte, le Cirad a été sollicité en 2006 afin d'œuvrer concrètement à la réhabilitation de l'arganeraie, sous la forme d'un projet financé pour une durée de trois ans. Le but de ce projet est d'améliorer, par la recherche appliquée, la qualité de l'arganeraie à travers la production d'arganiers de qualité supérieure issus d'individus sélectionnés. Ceux-ci seront multipliés en masse au moyen des techniques de clonage les plus adaptées, en tirant profit pour les sélections de la grande variabilité existant entre les plants issus de graines, pour produire des populations clonales plus homogènes. Après deux ans de collaboration avec le Centre Régional de Recherche Forestière de Marrakech et l'Université de Marrakech, plus d'un millier de boutures de jeunes arganiers ont pu être produites dans le cadre d'essais de mise au point de la technique de bouturage de l'espèce. Ce matériel sera utilisé comme pieds-mères expérimentaux et pour des tests de comportement au champ, en comparaison à des semis traditionnels. Par ailleurs, sur les quatorze têtes de clones sélectionnées par les populations locales, huit ont pu être mobilisées pour amorcer la propagation en masse par bouturage, avant les tests au champ. Les techniques de multiplication végétative développées pourront également être mises à profit pour la production en quantité de plants à partir des semis issus des fruits récoltés sur les têtes de clones précitées, présumées de qualité supérieure.

Mots-clés: Argania spinosa, arganeraie, amélioration, clone, greffage, bouturage, sélection, agroforesterie.

\section{VEGETATIVE PROPAGATION OF ARGAN TREE, ARGANIA SPINOSA IN MOROCCO: THE JOHN GOELET PROJECT}

Argan tree is a multipurpose arborescent species of great socio-economical interest for south-west Morocco. It is utilized for nutrition of people and livestock, as well as in medicine and in cosmetics. It protects against desertification and erosion while producing wood for various end-uses. The natural stands, in constant reduction since the 19th century, are more and more threatened by anthropomorphic pressures, hampering the natural regeneration of the species. Given this situation, Cirad was asked in 2006 to take part in a practical project, that was to be financially supported for 3 years, with a view to restoring argan tree natural stands. The goal of this project is to improve, through applied research approaches, the quality of the current argan tree stands. Taking advantage of the variation within the species, superior mother trees (plus trees) will be selected. These latter will be mass propagated using the most suitable cloning techniques to produce ultimately better adapted and more homogeneous clonal populations. After 2 years of close collaboration with the Centre Régional de Recherches Forestières de Marrakech and the Université de Marrakech, on testing different rooting conditions, one thousand rooted cuttings have been produced from juvenile argan tree seedlings. This material will be used as experimental stock plants and for field tests, in comparison with traditional seedlings. In addition, out of the 14 plus trees selected by the local people to be mass clonally produced, 8 have already been successfully mobilized in an ex situ clone bank for initiating their mass clonal propagation by rooted cuttings, prior to field testing and identification of superior clones for plantation establishment. The vegetative propagation techniques developed can also be used for large scale production of rooted cuttings from the seedlings derived from the fruits collected from the plus-trees, presumably of superior quality.

Keywords: Argania spinosa, argan tree stands, clone, improvement, grafting, propagation by cuttings, selection, agroforestry.

\section{MULTIPLICACIÓN VEGETATIVA DEL ARGÁN, ARGANIA SPINOSA, EN MARRUECOS: EL PROYECTO JOHN GOELET}

El argán es un árbol multipropósito de gran interés socioeconómico para el sudoeste marroquí. Se utiliza para la alimentación de los hombres y el ganado, en la medicina y la cosmética, al tiempo que permite luchar contra la desertificación y la erosión produciendo madera para distintos usos. Las masas naturales, en constante disminución desde el s. XIX, están amenazadas por diversas presiones antrópicas, cada vez más fuertes, que reducen la capacidad de regeneración natural de la especie. En este contexto, se solicitó en 2006 la intervención del CIRAD para trabajar específicamente en la rehabilitación del arganal a través de un proyecto financiado por un período de tiempo de tres años. El objetivo de este proyecto es mejorar, mediante la investigación aplicada, la calidad del arganal a través de la producción de arganes de calidad superior resultando de individuos seleccionados. Éstos se multiplicarán masivamente mediante las técnicas de clonación más adaptadas, sacando provecho para las selecciones de la gran variabilidad existente entre las plantas resultantes de semillas para lograr producir poblaciones clonales más homogéneas. Tras dos años de colaboración con el Centre Régional de Recherche Forestière de Marrakech y la Universidad de Marrakech, se pudieron producir más de mil estacas de arganes jóvenes en los ensayos de puesta a punto de la técnica de estaquillado de la especie. Este material se utilizará como plantas madre experimentales y para las pruebas de comportamiento en campo, comparándolo con los materiales tradicionales. Por otra parte, de las 14 cabezas de clones seleccionadas por las poblaciones locales, se pudieron movilizar 8 para empezar la propagación en masa por estaquillado, antes de las pruebas en campo. Las técnicas de multiplicación vegetativa desarrolladas también podrán aprovecharse para la producción cuantitativa de plantas a partir de semillas procedentes de frutos cosechados en las cabezas de clones antes mencionadas y que se supone que son de calidad superior.

Palabras clave: Argania spinosa, arganal, mejora, clon, injerto, estaquillado, selección, agroforestería. 


\section{Introduction}

La gestion raisonnée de l'eau devient une préoccupation majeure à l'échelle planétaire et les cultures économes en eau suscitent un intérêt croissant. Les zones arides et semi-arides sont plus particulièrement concernées. Cette situation a incité le Maroc à instaurer un « Plan Maroc Vert 2008 » visant à remplacer l'agriculture extensive traditionnelle par des cultures à meilleur rendement et plus économes en eau dans lesdites zones (MINISTÈRE DE L'AGRICULTURE ET DES PêCHES MARITIMES, 2008). L'accent est mis sur l'agroforesterie en mélange avec des espèces arborescentes à forte valeur ajoutée telles que l'olivier (Olea europaea L.) et l'arganier (Argania spinosa L. Skeels). Cette dernière connaît un intérêt croissant depuis quelques années en raison notamment d'un engouement international pour l'huile d'argan, au point d'initier un projet sur sa multiplication végétative, présenté dans cet article.

\section{L’arganier et la situation de l'arganeraie au Maroc}

L'arganier, seul représentant de la famille des Sapotacées en Afrique du Nord, est une espèce arborescente endémique du Sud-Ouest marocain, du bord de mer à 1 6001700 mètres d'altitude sur les versants sud du Haut Atlas occidental et sur l'Anti-Atlas (AlIfRIQUI, 2004). Des peuplements relictuels existent aussi dans le Rif (Boudy, 1950 ; M'HIRIT et al., 1998). Les arganiers sont des arbres épineux pour leur grande majorité, à troncs tortueux pouvant atteindre 8 à $10 \mathrm{~m}$ de haut, avec une longévité de 300 à 350 ans (Boudy, 1950). Une propension naturelle à rejeter de souche et à produire dans certaines situations des marcottes, voire de rares drageons (photos 1, 2, 3 et 4), incitent à penser que certains génotypes ont pu se perpétuer depuis bien plus longtemps. La cime plus ou moins grande et étalée peut présenter un port en parasol, plus rarement pleureur. Les feuilles alternes, simples ou regroupées sous forme de rosettes, sont persistantes et ne tombent qu'en cas de sécheresse prononcée. Les fruits (photos 5) ont un noyau très dur qui contient les amandes (encore appelées localement " amandons ») d'où est extraite l'huile d'argan.

La forte variabilité phénotypique observée reflète la grande diversité génétique de l'espèce (FERRADous et al., 1997) qui lui permet de résister à des conditions écologiques très contraignantes, notamment du point de vue de la sécheresse du sol. Cette essence thermophile et xérophile exige un climat doux, sans grande amplitude thermique, mais avec un degré hygrométrique relativement élevé qui caractérise la façade atlantique du Sud-Ouest marocain (ALIFRIQUI, 2004).

L'arganier est un arbre à usages multiples, et par là même de grand intérêt socio-économique pour le Sud-Ouest marocain. Il est utilisé pour l'alimentation des hommes (huile) et du bétail (feuilles, fleurs, pulpe du fruit, tourteau), en cosmétique (fruits, huile, feuilles vertes), et il permet de lutter contre la désertification et l'érosion tout en produisant du bois à diverses fins.

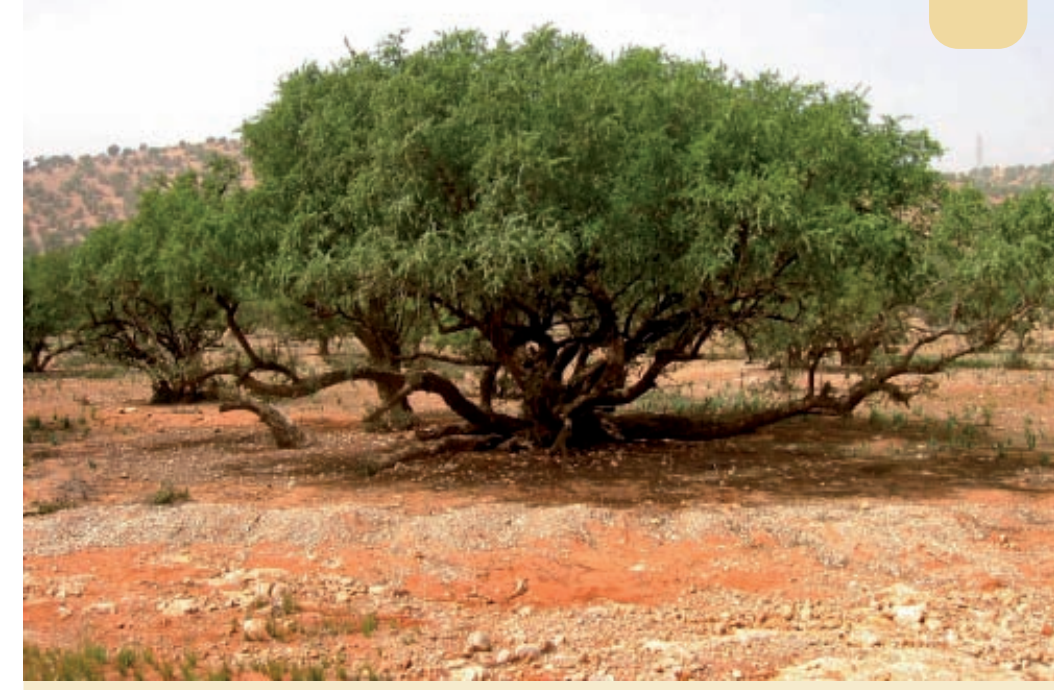

Photo 2.

Vieil arganier illustrant la propension naturelle à marcotter à partir de branches maîtresses basses. Photo O. Monteuuis.
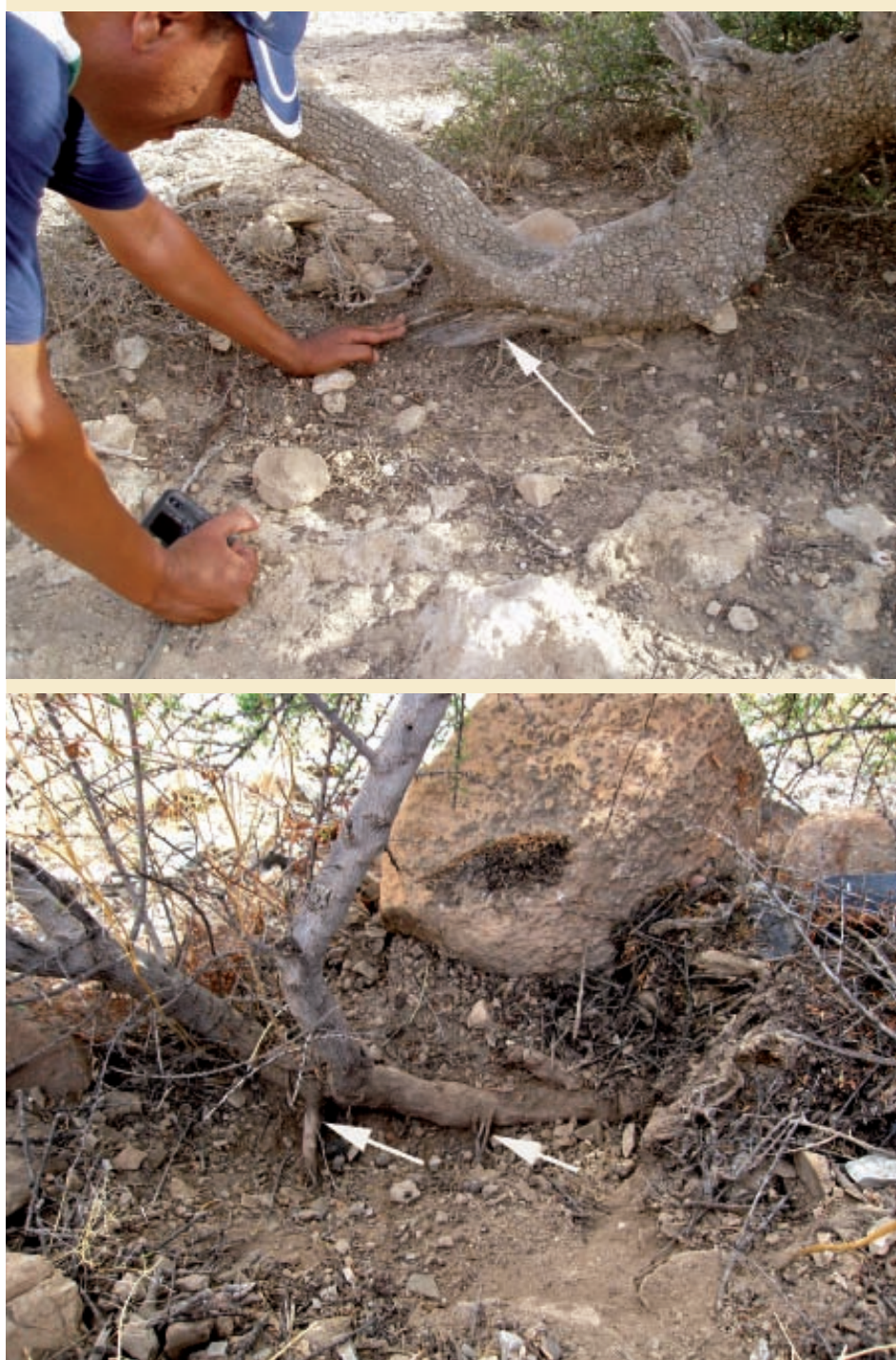

Photos 3.

Détails de marcottes naturelles plus jeunes montrant les racines adventives développées au contact du sol (flèches). Photo O. Monteuuis. 

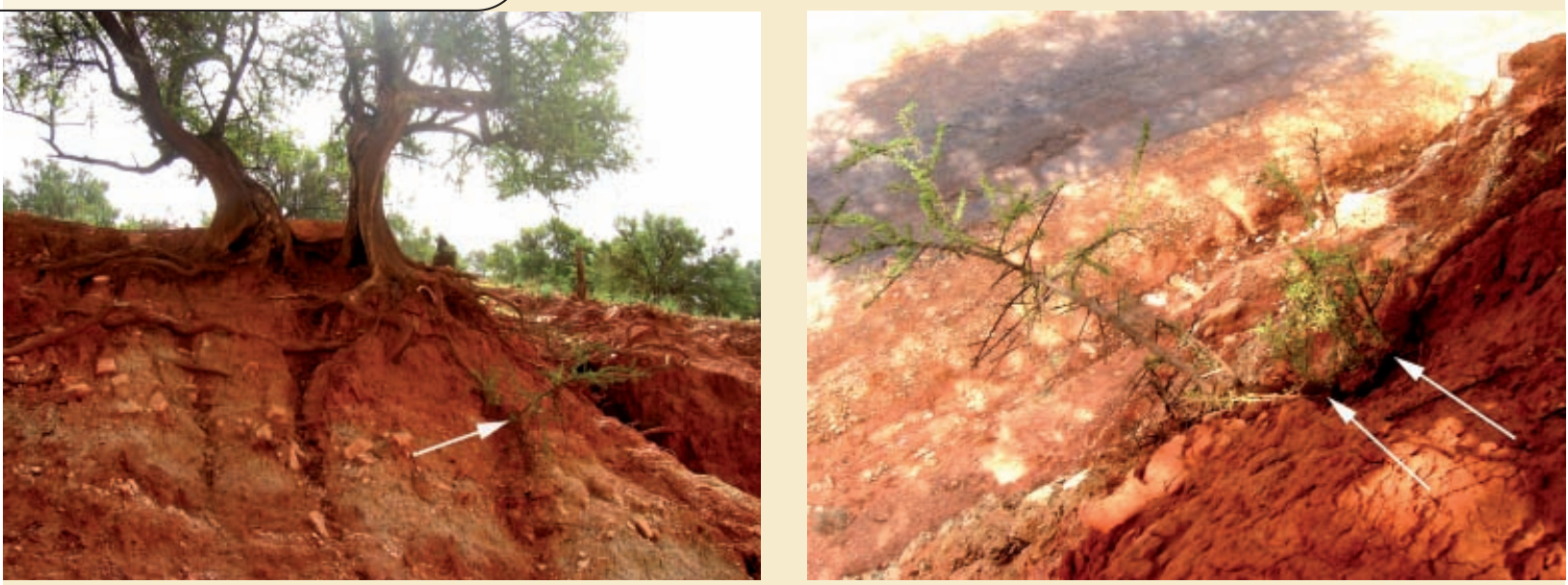

Photos 4

Certains individus peuvent émettre des drageons (flèches) à partir de racines mises à nu notamment.

Photo O. Monteuuis.

L’arganeraie est un écosystème dans lequel l'arganier occupe une place prépondérante. Elle constitue en superficie l'une des principales formations forestières du Maroc, couvrant, selon les sources, de 700000 (Boudy, 1950) à 828000 hectares (M'HIRIT et al., 1998), voire 870000 ha (KENNY, 2007), principalement localisés dans un triangle géographique limité sommairement au nord par Safi, à l'ouest par les massifs du Haut Atlas et le Djebel Sirroua et au sud par une ligne longeant l'Oued Noua au sud de Guelmim (figure 1). L'arganeraie de plaine, avec une densité moyenne de 100 souches à l'hectare, représente environ 10 à $15 \%$ de la superficie totale couverte par l'arganier, le reste correspondant à l'arganeraie de montagne dont la densité peut atteindre 700 à 800 souches par hectare et qui monte jusqu'à une altitude de 1400 m (M'HIRIT et al., 1998). L'arganeraie de plaine, surtout, est de plus en plus altérée par les pressions anthropiques liées à l'essor démographique, ce qui incite certains spécialistes à préférer la distinction entre l'arganeraie-verger et l'arganeraie-forêt (ALIFRIQUI, 2004).

L'arganeraie-verger, avec une densité moyenne de 100 souches par hectare, se rencontre généralement dans les zones de plaine, peu accidentées, fortement mises à profit pour l'agriculture, la céréaliculture plus spécialement (photo 6), et l'élevage responsable de surpâturage par le cheptel local, majoritairement représenté par les chèvres.

L'arganeraie-forêt subsiste dans les parties non cultivables du littoral atlantique (Essaouira, Agadir, Guelmim) et sur les contreforts montagneux au relief plus accidenté. Ces peuplements, dont la densité peut atteindre 500 souches par hectare, conservent une diversité infraspécifique plus élevée que l'arganeraie-verger.

Depuis le XIXe siècle, l'arganeraie subit une régression de plus en plus préoccupante sous les effets conjugués de plusieurs facteurs.

Ainsi, l'intensification de la production de charbon durant les années 1917-1924 pour l'approvisionnement des grands centres urbains de Marrakech, Safi et Casablanca a conduit à la destruction de 200000 ha. Quelques années plus tard, pour les besoins de la Seconde Guerre Mondiale, 40000 ha supplémentaires ont été exploités (ALIFRIQUI,
2004). Depuis lors, l'exploitation des arganiers comme source de bois de feu ou de service perdure, même parfois à partir de sujets vivants, en toute illégalité.

L'intensification de la céréaliculture, la mise en eau des périmètres irrigués du Souss et du Massa notamment, ainsi que l'introduction du maraîchage ont très fortement accentué les défriches, surtout en zones de plaine, plus accessibles et exploitables.

La surexploitation régulière du sous-bois des arganeraies, notamment des plantes accompagnatrices fixatrices d'azote, dont des Papillionacées, a entraîné une perte de fertilité des sols. Les pratiques aratoires « en plein » d'une agriculture en pleine expansion et les chèvres détruisent les rares germinations spontanées.

L'élevage caprin prédomine dans la région de l'arganeraie et les chèvres, de plus en plus nombreuses, ont la faculté de grimper dans les houppiers des arganiers pour brouter entre autres les jeunes pousses tendres et les fleurs (photo 7), le cas échéant aidées par les bergers (EL AICH et al., 2005). Elles peuvent être relayées par les troupeaux de dromadaires en provenance du Sud.

La diminution de l'arganeraie s'est accélérée avec la croissance démographique, l'expansion des villes et des terrains constructibles. L'exploitation des ressources halieutiques et le développement des sites touristiques de la zone littorale, surtout dans la périphérie d'Agadir, ont également contribué à la disparition de nombreux espaces à arganiers.

Depuis plusieurs années, l'engouement croissant à l'échelle internationale pour l'huile d'argan à des fins alimentaires, cosmétiques ou médicinales a entraîné, sous l'emprise de mouvements coopératifs et associatifs locaux, une très forte augmentation des récoltes de fruits. Ceux-ci sont pratiquement tous ramassés et il n'en subsiste donc au bout du compte que très peu susceptibles d'assurer la régénération naturelle des arganiers, phénomène rare au demeurant (M'HIRIT et al., 1998).

Enfin, la diminution constante des pluies depuis plusieurs années dans la zone de l'arganeraie, comme dans bon nombre d'autres régions du globe, contribue au dépérissement des arganiers en réduisant leur capacité à se régénérer naturellement par graines (photo 8). 

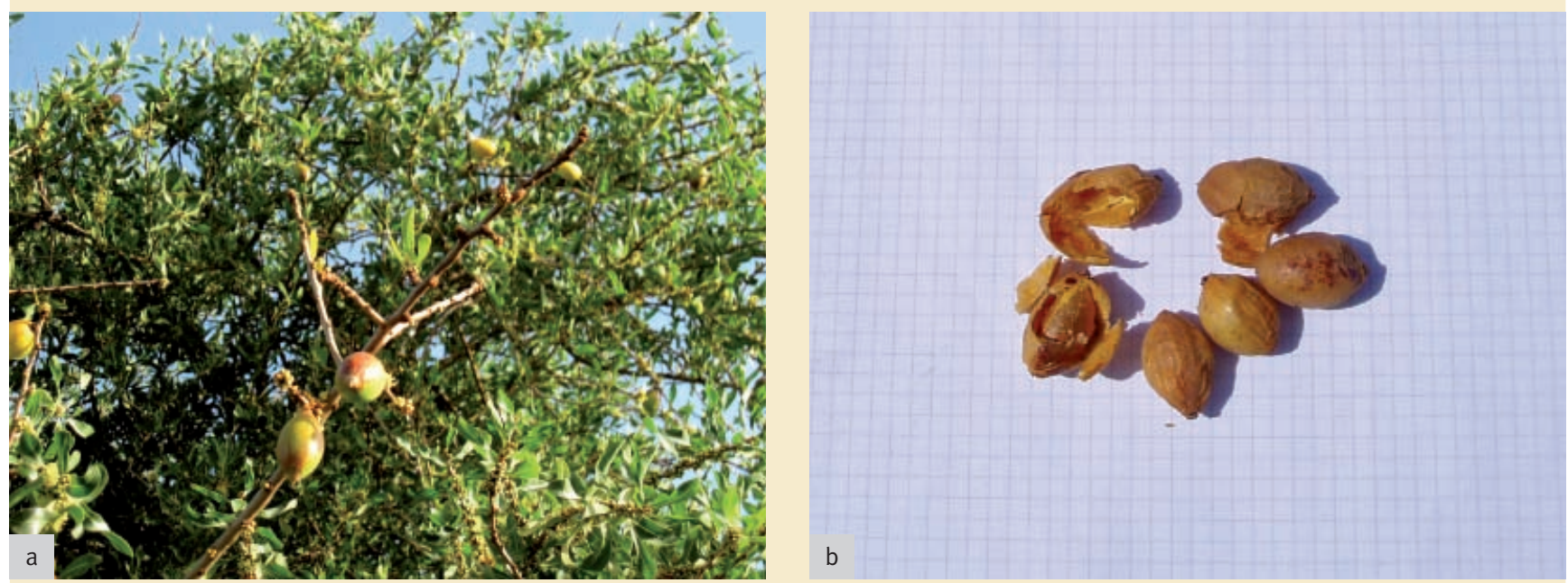

Photos 5.

Rameaux d'arganier portant des fruits d'où sont extraits les amandons (a) et fruits d'arganier de la variété Tablouht (b).

Photos O. Monteuuis (a), R. Bellefontaine (b).

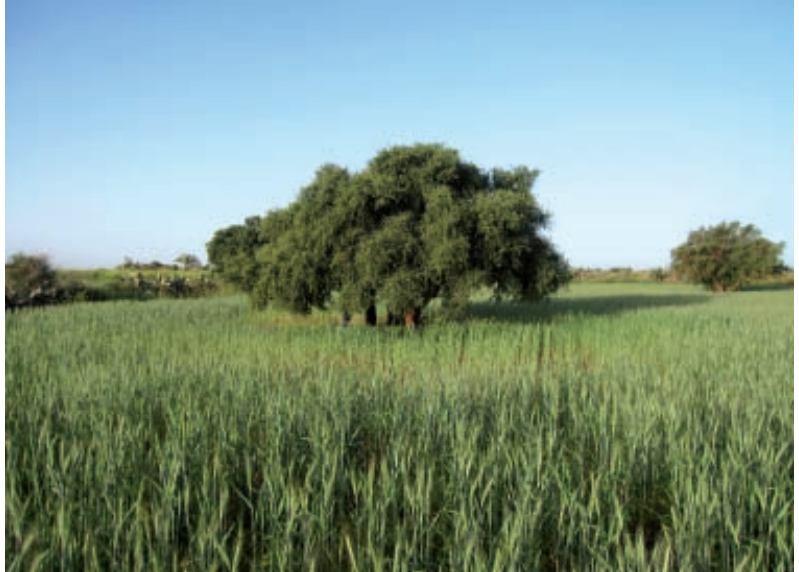

Photo 6.

Exemple d'arganeraie-verger avec culture de céréale (blé dans le cas présent) entre les rares arganiers subsistant. Photo R. Bellefontaine.

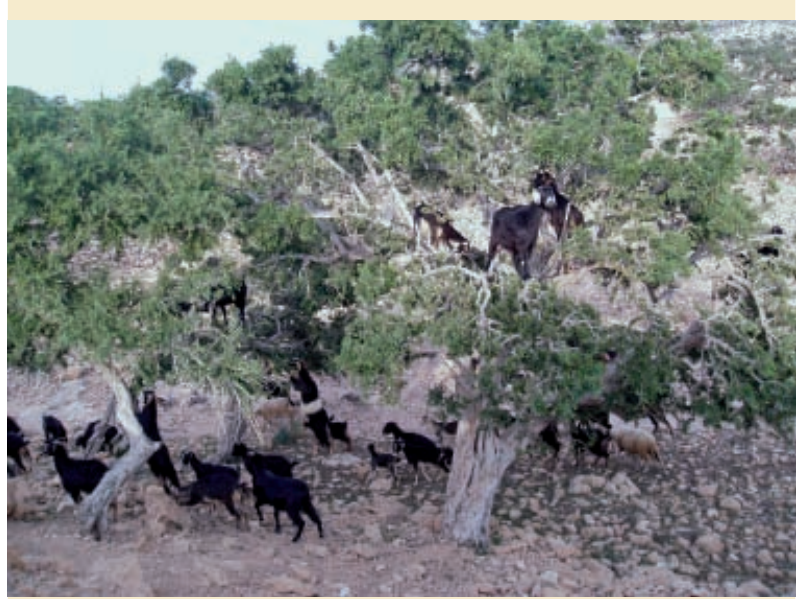

Photo 7.

Broutage des arganiers par les chèvres. Photo A. Galiana.

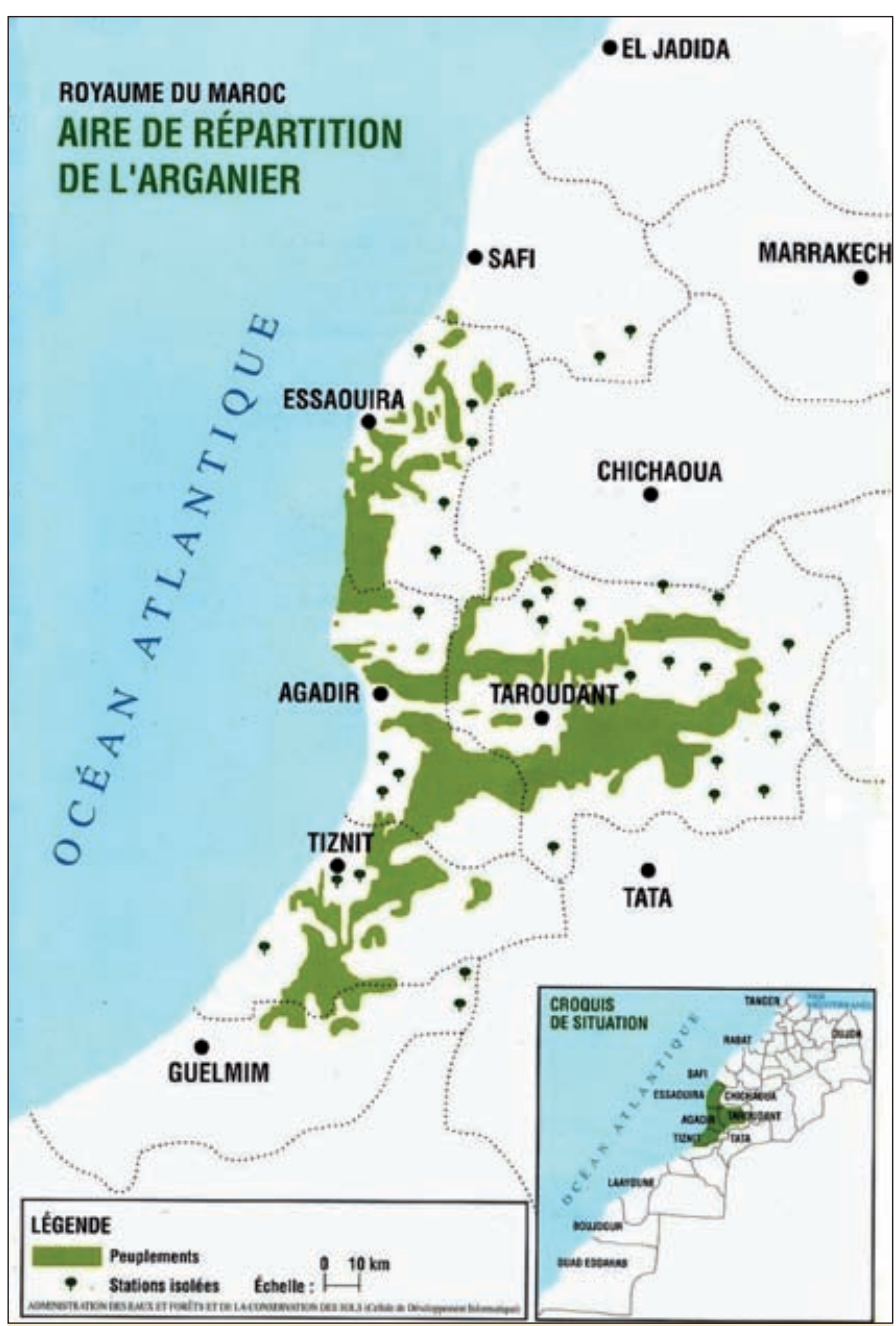

Figure 1.

Carte de l'aire de répartition de l'arganier dans le Sud-Ouest marocain (M'HIRIT et al., 1998). 


\section{Réhabilitation de l’arganeraie}

La réhabilitation de l'arganeraie se justifie donc par rapport à des fins écologiques, notamment pour contrer la désertification, économiques et socioculturelles évidentes. Actuellement, les opérations de reboisement et de protection de l'arganier se heurtent à une opposition quasi systématique de la part des populations qui n'acceptent pas facilement la nécessité des mises en défens d'une dizaine d'années, voire plus, qui accompagnent ces opérations. Les essais de plantations débutés il y a une trentaine d'années sous l'égide de la recherche forestière et des services forestiers (PLATTEBORZE, 1977) se sont soldés pour la plupart par des échecs (M'HIRIT et al., 1998 ; FerRAdous, 2008). Jusqu'en 2002-2003, la régénération de l'arganeraie de plaine s'est faite à une cadence trop lente pour compenser les pertes annuelles estimées en moyenne à 600 ha (EL Yousfl, 1988). Le bilan reste donc très largement déficitaire. Plus récemment, les techniques de semis en pépinière et de plantation ont été quelque peu améliorées (AlouAni, 2003 ; Alouani, BANI-AAmeUr, 2004 ; NouAIM, 2005 ; Ferradous, 2010), mais de sérieuses lacunes persistent.

Entre Tiznit et Tafraoute, dans le secteur de Tifadine, caractérisé par une pluviométrie inférieure à 200 millimètres par an, des semis issus de graines non sélectionnées (« tout venant ») sont produits dans des pépinières administratives locales en repiquant les jeunes germinations réalisées en planches dans des sacs de polyéthylène de 25 centimètres de haut, remplis de terre superficielle, technique quelque peu obsolète. Après 5 à 6 mois d'éducation-élevage au minimum, ces plants de 15 à $20 \mathrm{~cm}$ de haut sont plantés au champ en novembre-décembre, parfois en février-mars pour les années plus sèches. Mais trop souvent un « chignon » racinaire induit par les conteneurs en polyéthylène condamne leur avenir à plus ou moins longue échéance (FALCONNET et al., 2007). La densité est généralement de 200 individus par hectare $(7 \mathrm{~m}$ par $7 \mathrm{~m}$ ). Les trous de plantation, de 60 à $70 \mathrm{~cm}$ de profondeur en moyenne, sont réalisés mécaniquement, à l'aide d'un trac-

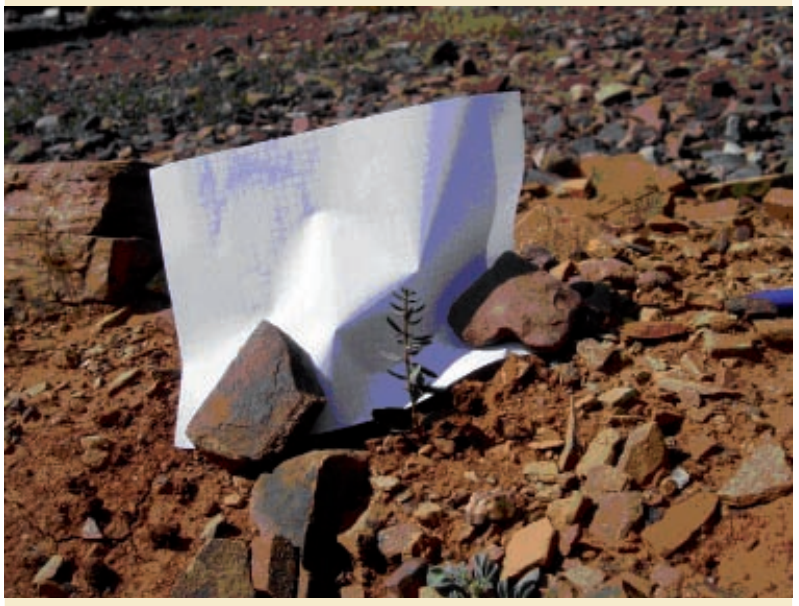

Photo 8.

Rare cas de régénération naturelle à Tifadine, dans la région de Tiznit, sous environ $175 \mathrm{~mm}$ de pluies par an.

Photo R. Bellefontaine.

teur équipé d'un godet, ou manuellement le plus souvent. Le plant débarrassé de son sachet est placé au sein d'une cuvette destinée à récolter le maximum d'eau de pluie, et dont le bord sud est plus haut de façon à protéger partiellement le jeune arganier du vent. Les plants sont arrosés deux à trois fois entre février et juin lors de la première année suivant la plantation, à raison de 10 litres par plant et par arrosage. Dans ce secteur de Tifadine, plus de 300 ha ont été ainsi reboisés depuis 2002. Le taux de reprise à un an est de $98 \%$ (photos 9). Il chute légèrement après la première année, mais en mai 2008 il était encore de l'ordre de 70 à $80 \%$ pour la plupart des parcelles. Les arganiers âgés de 4 à 5 ans atteignaient alors en moyenne 0,8 à 1,2 $\mathrm{m}$ de haut et le plus grand dépassait 2 m (BouICHE, 2008).

D'autres périmètres de replantation d'arganiers « tout venant » ont été réalisés sur plusieurs centaines d'hectares entre 2002 et 2008 (tableau I) dans les provinces dépendant
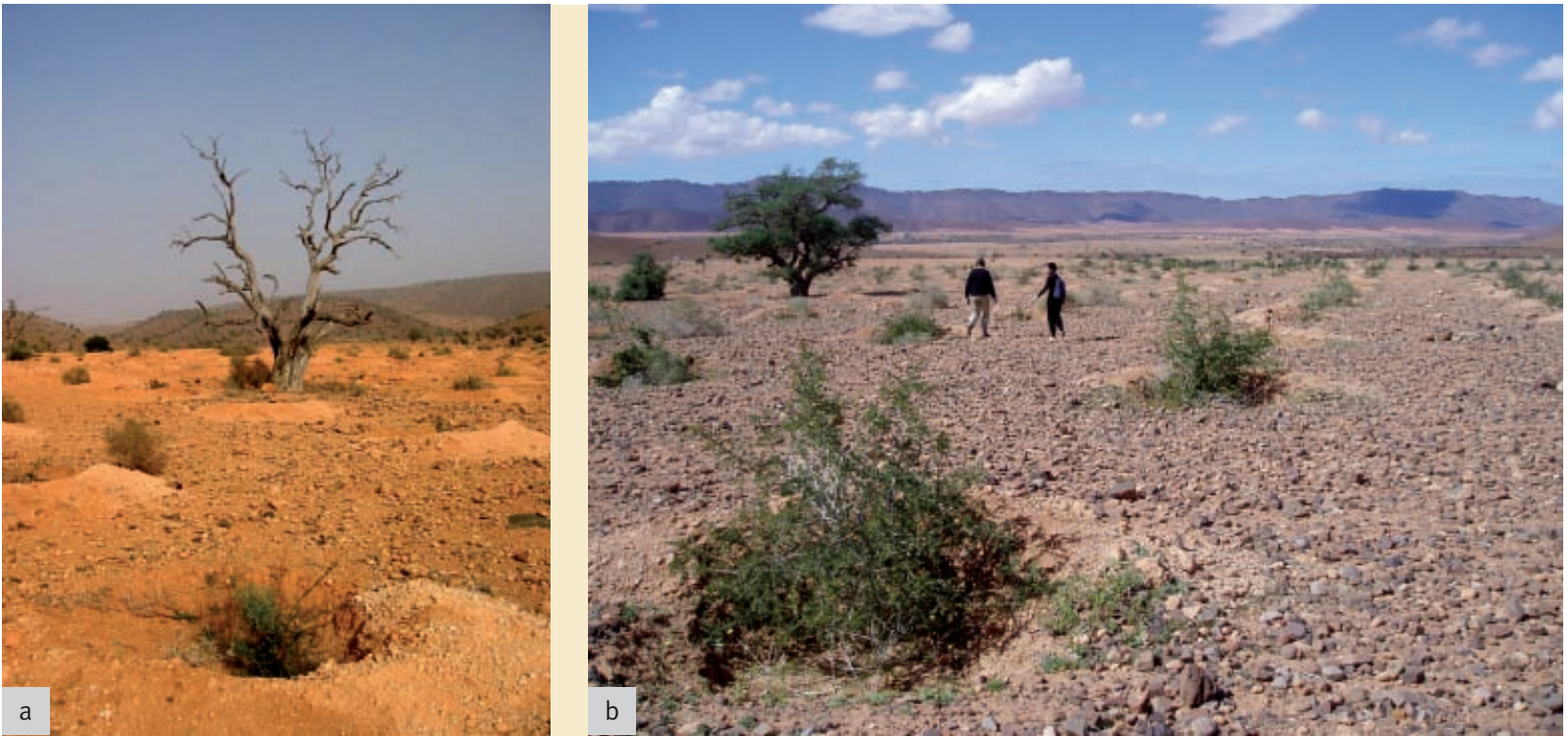

Photos 9.

Réhabilitation de l'arganeraie de plaine par plantation de semis en cuvette dans le secteur de Tifadine. Photos O. Monteuuis (a), R. Bellefontaine (b). 
des Directions régionales des Eaux et Forêts du Sud-Ouest et du Haut Atlas, avec des résultats variables et globalement inférieurs à ceux obtenus pour le secteur précité de Tifadine (selon un certain nombre d'observations des auteurs et de techniciens des Eaux et Forêts). Les techniques de pépinière, notamment du point de vue des conteneurs et substrats utilisés, ainsi que les phases d'éducation-élevage destinées à bien préparer les plants au transfert au champ dans des conditions bien plus éprouvantes qu'en pépinière, devraient être améliorées. De même, les arrosages effectués individuellement et de façon répétée pour chaque arganier nouvellement planté favorisent nettement la reprise.

Le Plan d'action du HCEFLCD ${ }^{1}$ de décembre 2007 mais surtout le tout récent «Plan Maroc Vert » présenté le 23 avril 2008 insistent sur la stratégie agricole et les nouvelles directives à adopter pour lutter contre la désertification (MINISTÈre de l'AgRICULTURE Et des PÊCHeS MARITIMES, 2008). Une restructuration profonde de l'économie agricole a été lancée début 2008. Ainsi, les cultures qui rapportent peu, au premier rang desquelles figurent notamment les céréales avec 2000 dirhams, soit environ 180 euros par hectare et par an, encore prépondérantes bien que très sensibles aux aléas de la sécheresse, doivent être remplacées par des cultures à revenu moins aléatoire. Les espèces à grande valeur ajoutée comme l'arganier sont judicieusement préconisées pour les régions arides.

\section{Présentation du projet}

Dans ce contexte, le Cirad ${ }^{2}$ a été sollicité en 2006 par Monsieur John GoELET afin d'œuvrer concrètement, sous la forme d'un projet financé pour une durée de trois ans, à la réhabilitation de l'arganeraie, avec ses effets bénéfiques tant au plan écologique, qu'économique et socioculturel.

\section{Objectifs et mise en place du projet}

Le but du projet financé par Monsieur John Goelet est d'améliorer, par la recherche appliquée, la qualité de l'arganeraie à travers la production d'arganiers de qualité supérieure issus de têtes de clone (" arbres plus »), sélectionnées, âgées et multipliées en masse au moyen des techniques de clonage les plus adaptées. À l'instar de nombreuses espèces et variétés fruitières, plus spécifiquement l'olivier dans le contexte local, le clonage de l'arganier peut se concevoir pour tirer profit de la grande variabilité existant entre les plants issus de graines, tout en produisant des populations clonales plus homogènes.

Les principaux bénéficiaires doivent être les populations locales vivant de l'arganeraie sous ses diverses formes, mais surtout de la production d'huile d'argan à forte valeur ajoutée. Les plantations résultantes contribueront à rajeunir et à réhabiliter l'arganeraie, tout en luttant contre la désertification, au profit de l'ensemble des habitants.

${ }^{1}$ Haut Commissariat aux Eaux et Forêts et à la Lutte Contre la Désertification. ${ }^{2}$ Centre de coopération internationale en recherche agronomique pour le développement.

Tableau I.

Superficies reboisées en arganier dans la zone de la DREF-SO*.

Années

Superficies (ha)

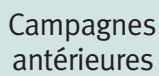

Campagnes antérieures

2002-2003

150
2003-2004

230

500

$2004-2005$

$2005-2006$

984

984

* Données de la Direction régionale des eaux et forêts du Sud-Ouest (Dref-SO). 
Concrètement, la réalisation de ce projet repose sur le choix des partenaires et acteurs marocains, principaux concernés, sur la sélection des têtes de clone et la mise au point des itinéraires techniques les plus adaptés aux objectifs.

L'Université de Marrakech et l'AAMHNM³ sont chargées du choix des terrains et des terroirs où sont sélectionnées les têtes de clone par les populations locales.

Le CRRFM ${ }^{4}$ a signé une convention avec l'Université afin de bénéficier des compétences disponibles dans le cadre de la mise en œuvre de projets communs. Par ailleurs, le CRRFM présente un certain nombre d'atouts justifiant son choix comme partenaire privilégié pour mettre en œuvre ce projet : spécificité, culture et compétences forestières, localisation pas trop éloignée des arganeraies naturelles en dépit de conditions climatiques bien différentes, motivation des responsables bien introduits au sein des communautés vivant de l'arganier et de ses produits dérivés. Le CRRFM dispose en outre de terrains, locaux, main-d'œuvre, moyens de communication ainsi que de l'approvisionnement permanent en eau et électricité avec des débits suffisants pouvant être mis à profit pour le projet.

La première tâche a été de conseiller et doter ce centre des équipements nécessaires au bouturage de l'arganier, pouvant aussi être utilisés pour d'autres espèces. Il s'agit en tout premier lieu d'installations de brumisation ou « mist system » de qualité. Le CRRFM a pu bénéficier rapidement d'installations de bouturage très satisfaisantes, avec un mist system diffusant, grâce à des automatismes programmables, de très fines gouttelettes d'eau qui restent quelque temps en suspension dans l'air. Cette qualité de l'aspersion permet d'augmenter les pulvérisations tant en durée qu'en fréquence, sans risquer des excès d'eau préjudiciables au substrat de bouturage. Ce système a été installé sous une ombrière à $60 \%$ d'ombrage. Les boutures sont placées en barquettes garnies de substrat de bouturage directement sous ce mist, ou protégées par un tunnel plastique refroidi

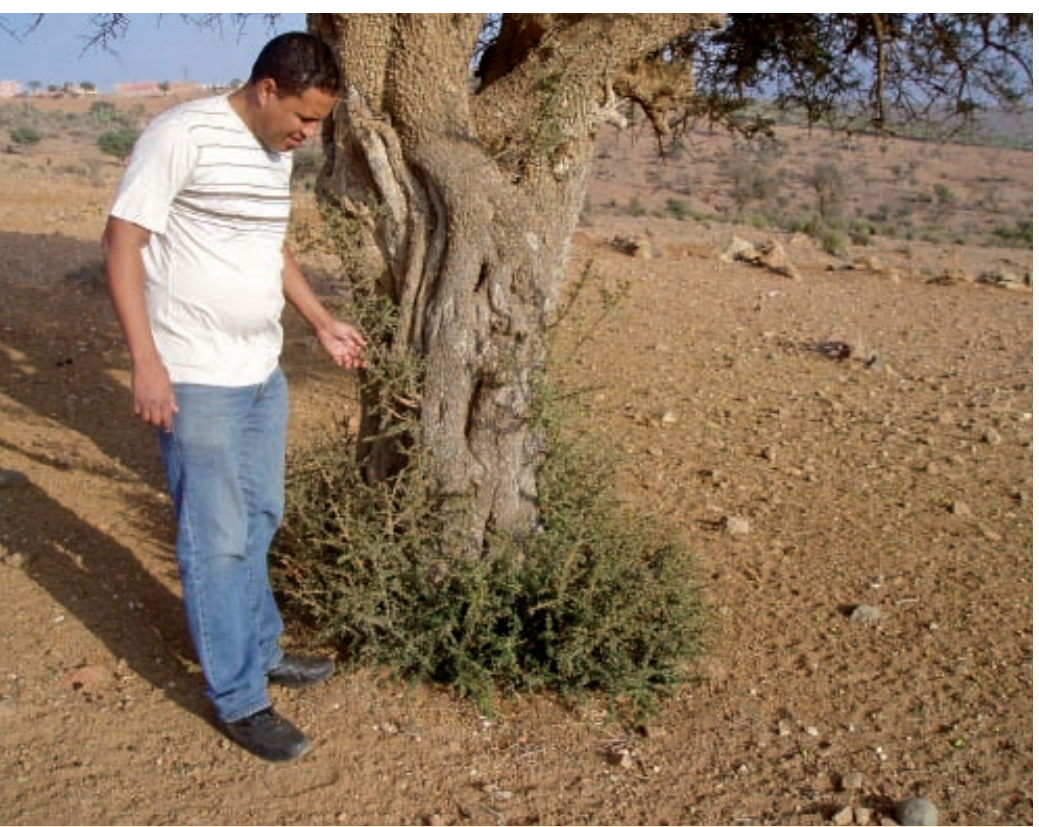

Photo 11.

Les rejets de base constituent un matériel prédestiné pour le bouturage.

Photo R. Bellefontaine. par le même mist (photo 10). Les programmateurs permettent de maintenir l'humidité relative requise en fonction des phases d'enracinement et de sevrage des boutures.

Le bouturage semble être en effet la technique la plus adaptée pour produire en quantité et à moindre coût des plants clonés à grande échelle, même s’il peut être nécessaire d'avoir recours à des techniques alternatives telles que le greffage et le marcottage aérien en aval pour mobiliser et rajeunir les têtes de clone sélectionnées âgées (MONTEUUIS, 1985, 1993 ; BeLLEFONTAINE, 2005 ; SAYA et al., 2008). Il importe malgré tout de tester dès que possible le comportement « au champ » de plants produits par bouturage par comparaison à des semis, en tenant compte des différences fondamentales d'appareil racinaire entre les deux origines, de type adventif pour les boutures contrairement aux semis.

La sélection des têtes de clone pour la production industrielle de plants d'arganiers clonés de qualité supérieure requiert un certain nombre de préalables. Il convient notamment de déterminer quels critères de sélection doivent être privilégiés, parmi plusieurs qui viennent tout naturellement à l'esprit : - rusticité et adaptabilité suffisantes des génotypes sélectionnés comme têtes de clone, donc à terme des clones produits ; - vigueur végétative assurant une croissance rapide durant les premières années du plant de façon à réduire la durée de mise en défens ;

- précocité d'entrée en fructification ;

- haute productivité annuelle en fruits, en tenant compte des variations possibles en fonction des années pour un même pied; - caractéristiques des fruits, noix, amandes et feuilles susceptibles d'optimiser leur utilisation pour l'homme et éventuellement le bétail, certains arganiers produisant des noix avec une coque qui se casse plus facilement, ce qui facilite le concassage (KAAYA, 1998) ;

- bons rendements en huile produite, en l'absence d'informations à ce jour quant à l’influence du génotype sur sa qualité.

L'idéal serait bien entendu de sélectionner comme têtes de clone les individus satisfaisant au mieux à l'ensemble de ces critères. Depuis quelques années, le principal attrait financier pour les populations locales reste la production d'huile d'argan, de grande valeur alimentaire et prisée également en cosmétique. En cohérence avec l'esprit et la finalité du projet, l'avis des exploitants a été privilégié quant au choix des meilleurs arganiers à cloner au sein de deux terroirs assez distants. Cette sélection s'est faite d'abord sur treize têtes de clone dans les régions d'Argana et de Smimou, en concertation avec les populations locales, sur la base initiale de la facilité de concassage manuel, des rendements en amandes et de la qualité de l'huile.

La région d'Argana est située à moins de 100 kilomètres $(\mathrm{km})$ au nord d'Agadir (figure 1). Du point de vue forestier, elle est gérée par le $\mathrm{SPEF}^{5}$ de Taroudant, qui dépend de la $\mathrm{DREF}^{6}$ du Sud-Ouest, dont le siège est situé à Agadir. Dans ce secteur, huit têtes de clone ont été sélectionnées dans des terrains privés sur la base de critères tels que la facilité de concassage des noix, le nombre d'amandes par fruit ou encore la qualité

\footnotetext{
${ }^{3}$ Association des Amis du Muséum d'Histoire Naturelle de Marrakech.

${ }^{4}$ Centre Régional de Recherche Forestière de Marrakech.

${ }^{5}$ Service provincial des eaux et forêts de Taroudant.

${ }^{6}$ Direction Régionale des Raux et Forêts.
} 
fourragère des fruits. Ces « arbres plus » ont la particularité de présenter de gros fruits les années suffisamment pluvieuses, avec une coque qui se casse aisément. Cette variété d'arganier, localement appelée " amrag » en berbère, est beaucoup plus prisée que la variété « adrdour » qui produit des noix à coque beaucoup plus difficile à casser et qui est délaissée lors des années très productives. Faute d' " amrag ", les récoltants les plus démunis se rabattent sur les " adrdour». Chacune des huit têtes de clone sélectionnées a été identifiée par un numéro de 1 à 8 peint en rouge sur deux faces du tronc, photographié et scrupuleusement localisé sur une carte avec les relevés de la position géographique à l'aide d'un appareil Gps (Global Positioning System). Des récoltes de pousses destinées à la multiplication végétative ont été effectuées à plusieurs reprises et périodes de l'année sur chacun de ces arganiers sélectionnés, préférentiellement dans la partie basale (photo 11), la plus accessible et présumée la plus apte au bouturage (MoNTEUUIS, 1985, 1998). Les fruits encore présents sur certaines de ces têtes de clone ont également été récoltés. L'identité et l'origine de ces matériels ont été scrupuleusement notées lors des récoltes, avant acheminement dans les plus brefs délais au CRRFM à des fins de multiplication.

Le second peuplement naturel d'arganiers retenu est situé à $45 \mathrm{~km}$ au sud d'Essaouira, dans la région de Smimou (SPEF d’Essaouira, voir figure 1). La présence de plusieurs variétés qui intéressent très directement les femmes chargées du concassage des noix, mais aussi les populations propriétaires de caprins, a été repérée. En plus des variétés qui se cassent facilement, appelées ici « tamrkhout », il existe des variétés précoces, " tamnzout », qui produisent des fruits bien avant les autres, au moment où l'offre est faible, pour un meilleur prix de vente de l'huile. La variété à coque très dure ou « tamaghlout » est ici aussi la moins prisée. Un arganier « tamrkhout » porte un second nom, « tablouht », quand il présente la particularité de fournir des fruits dont la chair (l'épicarpe et le mésocarpe) peut se détacher aisément, une fois séchée, en un ou deux morceaux et être conservée plus d'un an (photos 5). Contrairement à la plupart des fruits dont la chair sèche se fissure, puis se désagrège rapidement en petits morceaux et qui ne peut donc être récoltée, ces « tablouht » peuvent générer des réserves alimentaires pour le bétail en cas de disette.

Des rameaux et des fruits ont été récoltés selon la même procédure sur cinq nouvelles têtes de clone de ce second site, identifiées et numérotées de 9 à 13, toutes de la variété " tamrkhout » et de type précoce " tamnzout » pour les numéros 10 et 11 , et « tablouht » pour la tête de clone $n^{\circ} 13$.

Une dernière tête de clone, $n^{\circ} 14$, a été sélectionnée beaucoup plus récemment (début 2009) au sud d’Agadir, car présentant une vigueur et une productivité en noix remarquables avec deux fructifications par an. En sus d'être " remontant », cet arganier est inerme et produit des noix à coque fine, qui se cassent aisément pour extraire de grosses amandes.

Il nous a paru judicieux de limiter notre projet à ces 14 têtes de clone, effectif largement suffisant pour étudier sérieusement les capacités au clonage de l'arganier dans le cadre expérimental fixé.

\section{État d'avancement après deux années et perspectives}

Dès que les installations de pépinière et plus particulièrement le mist system ont été opérationnels, des jeunes semis non sélectionnés d'arganier ont été utilisés pour définir les meilleures conditions de bouturage, encore méconnues pour cette espèce. Les paramètres étudiés ont été classiquement : les substrats de bouturage, les traitements auxiniques exogènes (" hormones ») de la base des boutures, les ambiances de bouturage, à savoir sous confinement ou en plein air sous ombrière, et les effets saisonniers. Ces derniers, trop souvent sous-estimés (MoNTEUUIS, 1985 ; TEKLEHAIMANOT et al., 2004), sont susceptibles d'influencer très fortement l'aptitude au bouturage du matériel végétal au moment de la récolte à partir de la tête de clone ou du pied-mère, puis tout au long de la phase d'enracinement dans les conditions du CRRFM.

De nombreux essais ont été répétés depuis décembre 2007 de façon à optimiser les conditions de bouturage, du moins en ce qui concerne les facteurs exogènes précités, sans sous-estimer leurs interactions. Ces essais ont porté sur les semis " tout venant " évoqués précédemment, disponibles au sein du CRRFM, mais aussi sur l'ensemble des 14 têtes de clone sélectionnées in situ. Ils ont permis, à l'issue des deux premières années, de dresser le bilan suivant: - sur un total de 2490 boutures de semis «tout venant» mises en place pour tester les différentes modalités de bouturage, 1467 ont pu être enracinées et 1020 sevrées, ce qui correspond à des taux moyens d'enracinement et de sevrage de $58,9 \%$ et de $69,5 \%$ respectivement, et un taux de réussite global de $41 \%$ (photo 12 ) ; c'est la première fois que des boutures d'arganier, actuellement en phase d'élevage-éducation au CRRFM, sont produites en si grand nombre ;

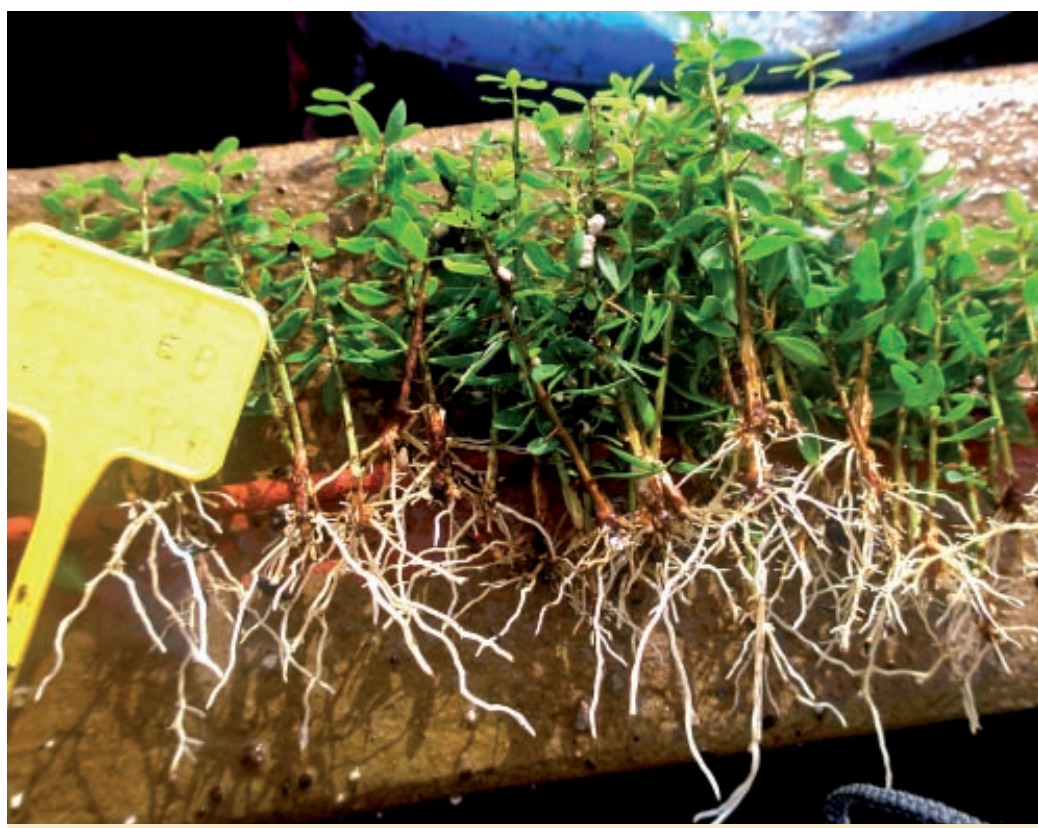

Photo 12.

Boutures enracinées de jeunes plants d'arganier. Photo A. Ferradous. 
Tableau II.

Inventaire en septembre 2009 des clones d'arganier sélectionnés âgés.

\begin{tabular}{|c|c|c|c|c|c|c|c|c|c|c|c|c|c|c|}
\hline Clones & 1 & 2 & 3 & 4 & 5 & 6 & 7 & 8 & 9 & 10 & 11 & 12 & 13 & 14 \\
\hline $\begin{array}{l}\text { Nombre de } \\
\text { boutures effectuées }\end{array}$ & 183 & 186 & 234 & 120 & 217 & 41 & 119 & 246 & 377 & 306 & 342 & 307 & 317 & 69 \\
\hline $\begin{array}{l}\text { Nombre de } \\
\text { boutures réussies }\end{array}$ & 0 & 0 & 0 & 0 & 2 & 0 & 0 & 1 & 0 & 2 & 0 & 2 & 4 & 29 \\
\hline $\begin{array}{l}\text { Nombre de } \\
\text { greffes effectuées }\end{array}$ & 77 & 72 & 72 & 72 & 72 & 77 & 77 & 72 & 92 & 97 & 97 & 92 & 97 & 25 \\
\hline $\begin{array}{l}\text { Nombre de } \\
\text { greffes réussies }\end{array}$ & 0 & 1 & 1 & 2 & 2 & 0 & 1 & 2 & 1 & 5 & 0 & 1 & 0 & 0 \\
\hline $\begin{array}{l}\text { Effectif encore en vie } \\
\text { en septembre } 2009\end{array}$ & 0 & 0 & 1 & 2 & 4 & 0 & 0 & 2 & 0 & 6 & 0 & 1 & 1 & 19 \\
\hline
\end{tabular}

- le matériel plus âgé prélevé sur les individus plusieurs fois centenaires sélectionnés s'est montré, sans surprise (MoNTEUUIS, 1985, 1993), beaucoup moins réactif (tableau II), et le greffage a été pratiqué conjointement au bouturage pour chacune des 12 récoltes effectuées jusqu'alors. La technique « en tête » a été préférée (photos 13), parfois couplée à la technique " en lanière ", encore appelée " en placage ", de façon à économiser les porte-greffes de semis (CHAMPAGNAT, 1980 ; MONTEUUIS, 1985 ; HARTMANN et al., 1997).

L'inventaire réalisé en septembre 2009 dénombrait, tous prélèvements et clones confondus, 3064 boutures mises à enraciner et 1091 greffes effectuées depuis le début du projet. La faible réactivité au bouturage constatée confirme pour l'arganier, comme pour la plupart des espèces arborescentes, que l'aptitude à néoformer des racines adventives décroît considérablement avec l'âge des sujets (MonteuUis, 1985, 1993 ; SAYA et al., 2008). En ce qui concerne le greffage, à l'instar d'autres espèces, une faible aptitude spécifique au type de greffe pratiqué, voire au greffage plus généralement, peut être la cause de ces résultats jusqu'à présent décevants (MoNTEUUIS, 1995). Les écarts climatiques très contrastés (spécialement en hiver) entre Marrakech et les sites d'origine peuvent entraîner des incompatibilités physiologiques entre l'activité des porte-greffes et celle des greffons (ChAMPAGNAT, 1980 ; HARTMANn et al., 1997), difficiles à déterminer de visu. Par ailleurs, les types de pousses utilisables comme greffons varient considérablement d'une tête de clone à une autre, de même que l'activité physiologique de ces pousses au sein d'un même arbre. Ainsi, l'écussonnage, déjà tenté avec succès mais sur un échantillon trop restreint à ce jour pour être significatif, a pu être pratiqué à plus grande échelle seulement lors de la dernière récolte, à partir du seul individu présentant les prédispositions requises pour ce type particulier de greffe (photos 13).
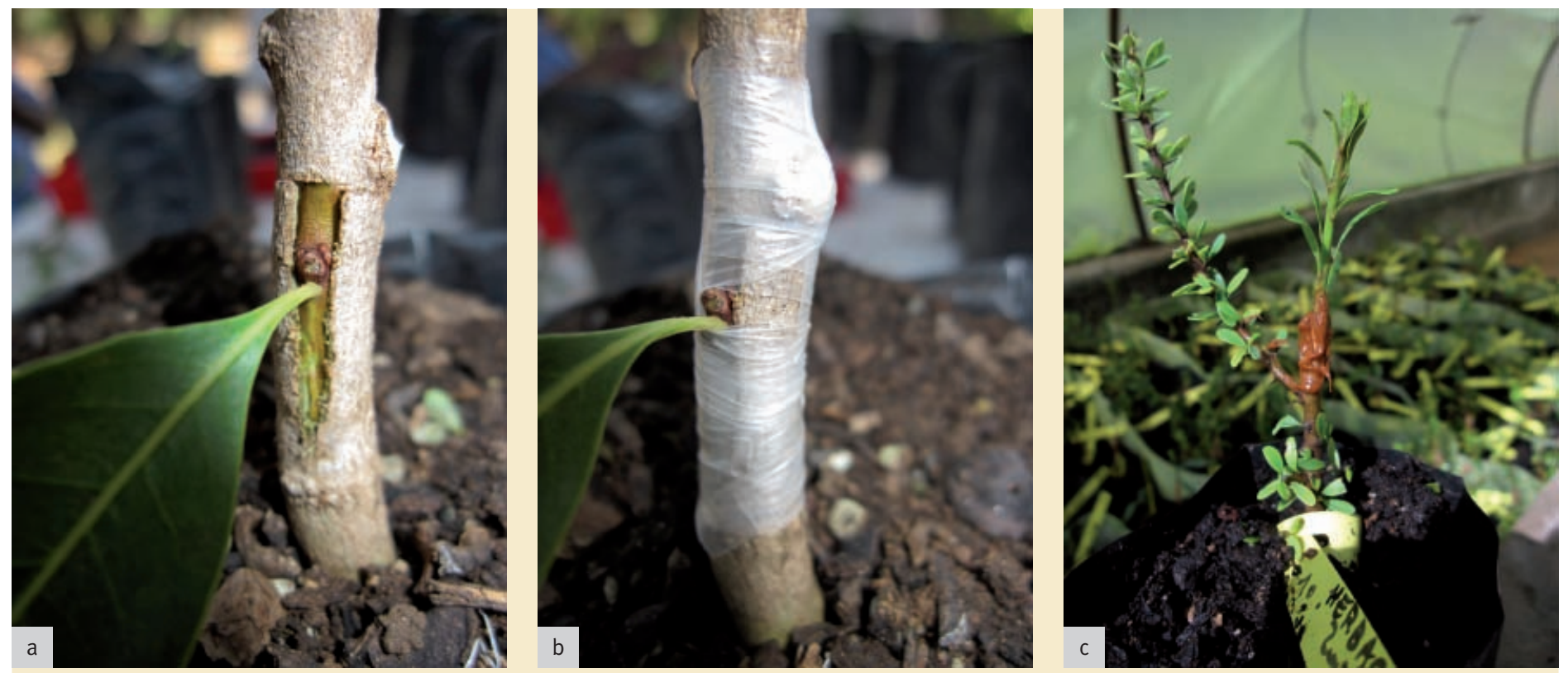

Photos 13.

Mobilisation de génotypes sélectionnés âgés par greffage en écusson avant et après ligature (a et b), et en fente terminale (c). Photo O. Monteuuis. 
Par ailleurs, des pertes parfois sérieuses, notamment durant la saison chaude, sont à déplorer à l'issue du rempotage, après un sevrage réussi, donnant lieu à l'inventaire détaillé dans le tableau II, toutes saisons confondues.

Des effets clonaux semblent se dégager, au profit notamment du dernier clone sélectionné sous le numéro 14 . D'ores et déjà, nous disposons en pépinière de huit génotypes issus des trois zones de sélection in situ. Les efforts pour mobiliser les têtes de clone récalcitrantes se poursuivent, en envisageant le recours à d'autres techniques de mobilisation telles que le marcottage aérien (MONTEUUIS, 1985 ; HARTMANn et al., 1997 ; MEUnIER et al., 2008 ; photos 14), alors que des premières boutures plus réactives sont récoltées à partir des pieds-mères des clones 4,5 , 10 et 14, déjà mobilisés.

Les boutures de semis « tout venant » réussies seront utilisées à deux fins (figure 2) : - pieds-mères gérés de façon intensive en conteneurs hors-sol afin d'acquérir le savoir- faire nécessaire pour bouturer avec la meilleure efficience possible les premières boutures ou greffes réussies à partir des clones sélectionnés, conformément aux pratiques ayant fait leurs preuves sur de nombreuses autres espèces arborescentes (MONTEUUIS, 1985, 1993 ; SAYA et al., 2008) ; - plantations en tests comparatifs avec des semis "tout venant", de même développement ou âge, de façon à observer le comportement au champ de ces premières boutures d'arganier, en ce qui concerne la stabilité, la conformité de croissance tant racinaire qu'aérienne, la productivité en fruits, etc. Autant d'informations inconnues jusqu'à présent et pourtant déterminantes pour l'avenir commercial des boutures clonées d'arganier.
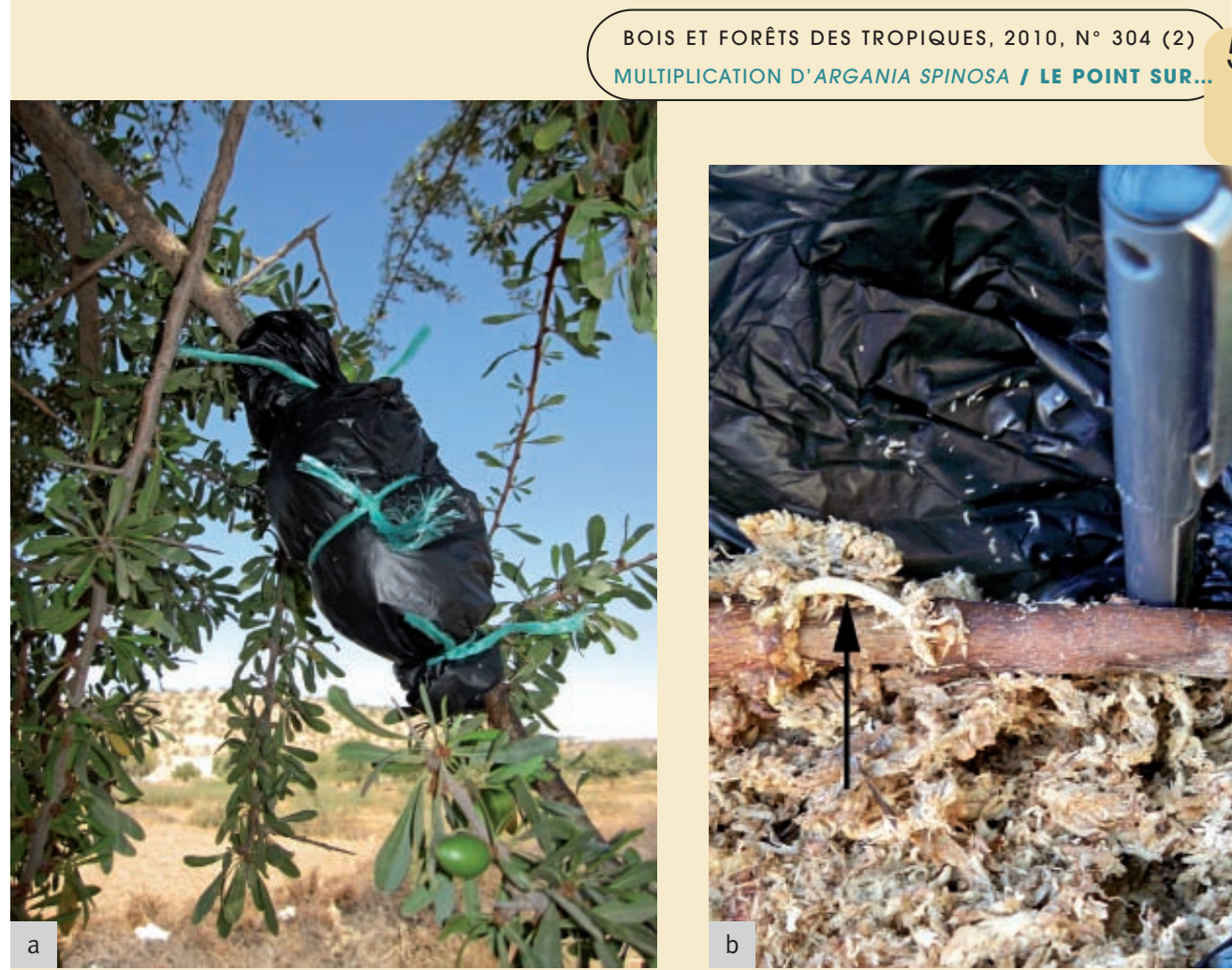

Photos 14

Marcotte aérienne juste après la mise en place afin de mobiliser un sujet « Plus » âgé (a) et quelques mois plus tard avec l'apparition des premières racines (b, flèche).

Photos O. Monteuuis (a) et A. Ferradous (b).

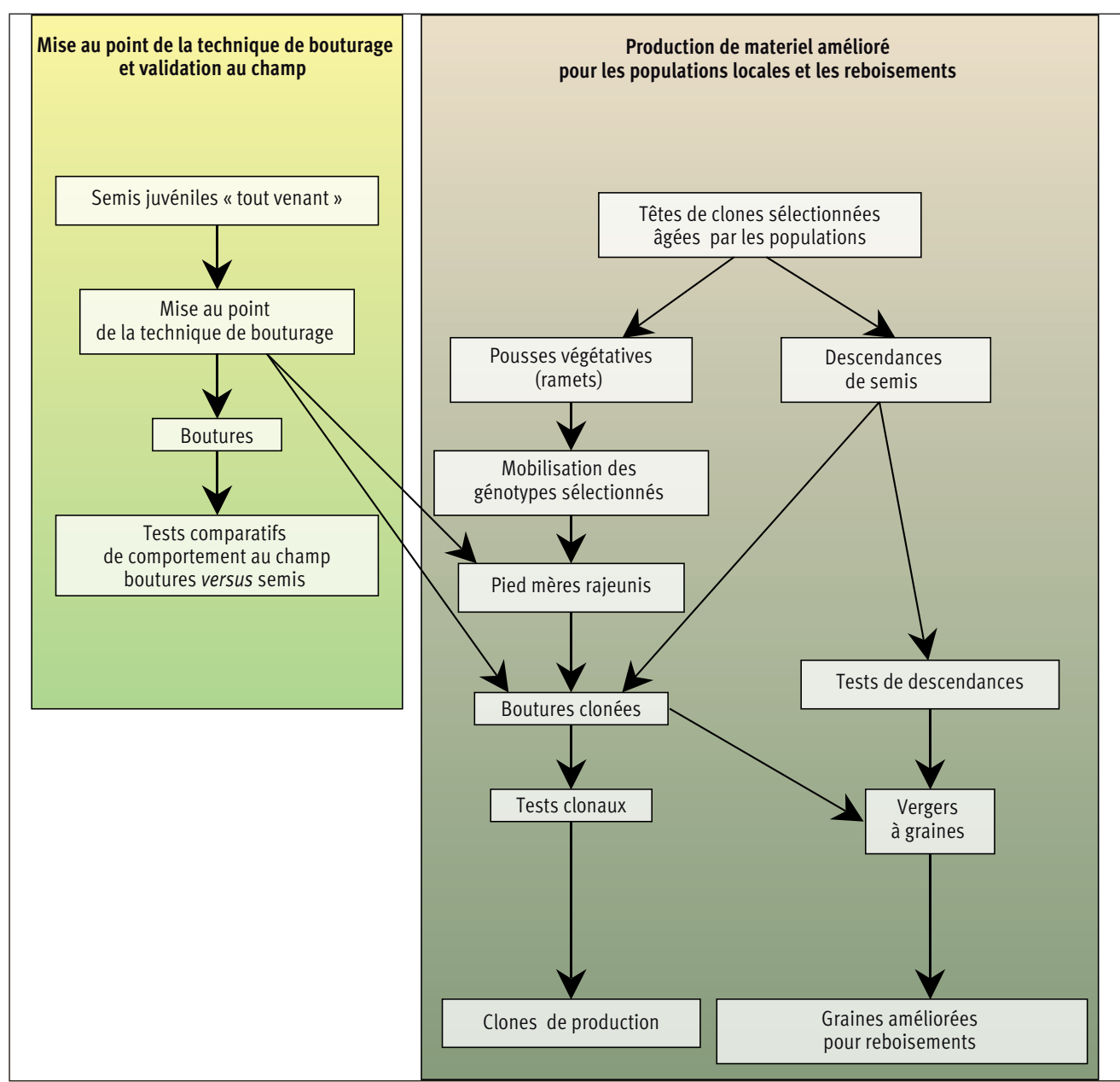

Figure 2.

Récapitulatif des différentes composantes du projet. 


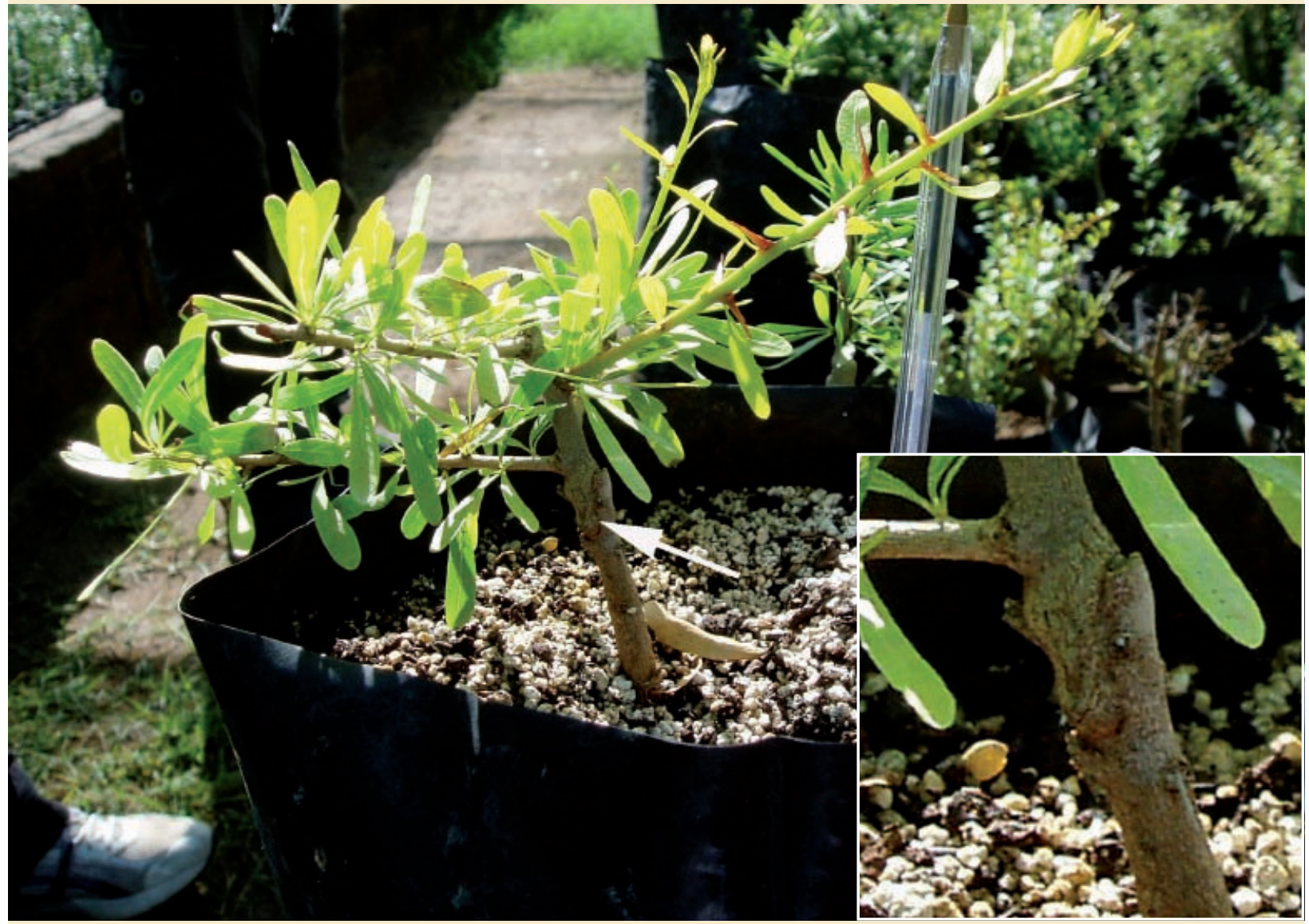

Photos 15.

Greffe en tête réussie (détail du point de greffe indiqué par la flèche dans l'encadré) d'une tête de clone âgée destinée à servir de pied-mère pour commencer le bouturage " en cascade ». Photos O. Monteuuis.

Les premiers représentants clonaux obtenus par greffage ou bouturage des 14 têtes de clone sélectionnées (photos 15) seront eux exclusivement destinés à un usage de pieds-mères gérés le plus judicieusement possible, en tirant enseignement de l'expérience acquise sur les boutures de semis « tout venant» et sur d'autres espèces (MonTEUUIS, 1993 ; SAYA et al., 2008). Le but est de parvenir le plus rapidement possible au rajeunissement physiologique requis pour obtenir des taux d'enracinement des boutures compatibles avec une production industrielle. À cette fin, le bouturage en cascade paraît être préconisé (MoNTEUUIS, 1993 ; SAYA et al., 2008), en s'affranchissant le plus tôt possible des pieds-mères obtenus par greffage, en raison des risques de bouturer les pousses illégitimes issues des portegreffes de semis non sélectionnés (et non du greffon).

Les premières boutures rajeunies obtenues seront fournies aux propriétaires des têtes de clone, bien légitimement, pour observation, avant de considérer les modalités d'une diffusion commerciale à plus grande échelle si nécessaire ou d'un verger à graines (figure 2).
L'intérêt des semis issus des fruits récoltés sur les têtes de clone sélectionnées se justifie à plusieurs titres :

- mise en place sur le terrain selon des dispositifs appropriés pour estimer le déterminisme génétique (héritabilité) des caractères les plus prisés chez l'arganier ; ces essais pourront ensuite être convertis en vergers à graines destinés à produire en grandes quantités des semis d'arganier de qualité supérieure pour les reboisements, en tirant profit de la bonne faculté de germination de l'espèce (AlouANI, 2003 ; NouAIM, 2005) ;

- production de pieds-mères juvéniles pour comparer le comportement au bouturage et au champ des boutures produites à partir de ces derniers et des arbres-mères sélectionnés âgés étroitement apparentés, donc en minimisant les biais d'ordre génétique. Ces pieds-mères pourront être utilisés pour la production de boutures clonées ou en mélange («bulk»), de façon à pallier l'insuffisance éventuelle de semis de la même origine pour les plantations de rapport. Ce matériel peut permettre également d'approfondir les connaissances quant à l'héritabilité des caractères de l'arganier, également méconnue. 


\section{Références bibliographiques}

ALIFRIQUI M., 2004. L'écosystème de l'arganier. Étude réalisée pour le Programme des Nations unies pour le développement (Pnud-Maroc), 126 p.

ALOUANI M., 2003. Régénération de l'arganier (Argania spinosa (L.) Skeels) : protocole de production de plants par semis et par bouturage et réussite de la transplantation. Thèse, Université Ibn Zohr, Faculté des sciences, Agadir, Maroc, $188 \mathrm{p}$.

ALOUANI M., BANI-AAMEUR F., 2004. Argan (Argania spinosa (L.) Skeels) seed germination under nursery conditions : effect of cold storage, gibberellic acid and mother-tree genotype. Annals of Forest Science, 61 (2) : 191-194.

BELLEFONTAINE R., 2005. Pour de nombreux ligneux, la reproduction sexuée n'est pas la seule voie : analyse de 875 cas. Sécheresse, 16 (4) : 315-317.

BOUDY P., 1950. Monographie et traitement de l'arganier. Dans Monographie et traitements des essences forestières. Paris, France, Éd. Larose, tome II, fascicule I, p. 382-416.

BOUICHE L., 2008. Étude des modes de régénération à faible coût de l'arganier (Argania spinosa) au Maroc. Master II Bioressources en régions tropicales et méditerranéennes, Université Paris XII, France, 60 p.

CHAMPAGNAT P., 1980. La greffe végétale. Dans Chaussat R., Bigot C. (éd.). La multiplication végétative des plantes supérieures. Paris, France, Gauthier-Villars, p. 99-114.

EL AICH A., BOURBOUZE A., MORAND-FEHR P., 2005. La chèvre dans l'arganeraie. Rabat, Maroc, Actes Éditions, 126 p.

EL YOUSFI S. M., 1988. Dégradation forestière dans le SudOuest, exemple de l'arganeraie d'Admine entre 1969 et 1986. Mémoire de $3^{e}$ cycle, Institut agronomique et vétérinaire Hassan II, Rabat, Maroc, 117 p.

FALCONNET G., HELDERLÉ C., BADINIER C., FERNANDEZ OSUNA J., 2007. Étude de l'incidence de la qualité des plants forestiers sur la pérennité des boisements et reboisements en Alsace. Installation de placettes permanentes et première série de mesures. Ministère de l'Agriculture et de la Pêche et Engref (France), 42 p. et annexes.

FERRADOUS A., BANI-AAMEUR F., DUPUIS P., 1997. Diversité génétique du fruit et de la graine de l'arganier. Dans Birouk A., Rejdali M. (éd.). Actes du séminaire sur les ressources phytogénétiques et le développement durable organisé à Rabat par le Comité national des ressources phytogénétiques. Rabat, Maroc, Actes Éditions, p. 319-324.

FERRADOUS A., 2008. Essais de provenances et tests de descendances chez l'arganier. Dans Actes des Premières Assises de la recherche forestière sur l'arganier, Essaouira, Maroc, 23-24 mars 2006. Rabat, Maroc, Éditions du Centre de la recherche forestière, p. 92-105.

FERRADOUS A., 2010. Production de plants d'arganier en pépinière. Fiche technique. Rabat, Maroc, Éditions du Centre de recherche forestière, $23 \mathrm{p}$. (sous presse).

HARTMANN H. T., KESTER D. E., DAVIES Jr F. T., GENEVE R. L., 1997. Plant propagation : principles and practices. Sixth edition. Upper Saddle River, New Jersey, États-Unis, Prentice Hall International, $770 \mathrm{p}$.
KAAYA M., 1998. Contribution à la domestication de l'arganier : sélection et multiplication. Thèse, Université Ibn Zohr, Agadir, Maroc, $175 \mathrm{p}$.

KENNY L., 2007. Atlas de l'arganier et de l'arganeraie. Agadir, Maroc, Institut agronomique et vétérinaire Hassan II, 191 p. MEUNIER Q., BELLEFONTAINE R., MONTEUUIS O., 2008. La multiplication végétative d'arbres et arbustes médicinaux au bénéfice des communautés rurales d'Ouganda. Bois et forêts des tropiques, 295 : 71-82.

M'HIRIT O., BENZYANE M., BENCHEKROUN F., EL YOUSFI S. M., BENDAANOUN M., 1998. L'arganier. Une espèce fruitière-forestière à usages multiples. Sprimont, Belgique, Éditions Mardaga, $151 \mathrm{p}$.

MINISTÈRE DE L'AGRICULTURE ET DES PÊCHES MARITIMES, 2008. Le «Plan Maroc Vert 2008 ». Rabat, Maroc.

MONTEUUIS O., 1985. La multiplication végétative du séquoia géant en vue du clonage. Annales de recherches sylvicoles 1984 (Afocel), p. 139-171.

MONTEUUIS O., 1993. Current advances in clonal propagation methods of some indigenous timber species in Sabah (Malaysia). In : Davidson J. (éd.). Recent advances in mass clonal multiplication of forest trees for plantation programmes. Proc. UNDP/FAO Regional Project on improved productivity of manmade forests through application of technological advances in tree breeding and propagation (FORTIP), Cisarua, Bogor, Indonesia, 1-8 Dec. 1992. Rome, Italie, Fao, p. 168-193.

MONTEUUIS 0., 1995. In vivo grafting and in vitro micrografting of Acacia mangium : impact of ortet age. Silvae Genetica, 44 (4) : 190-193.

MONTEUUIS O., 1998. Influence de la position in situ de la bouture sur son enracinement et son développement ultérieur: synthèse de travaux publiés. Dans Verger M., Le Pichon C., Le Bouler H. (éd.). Multiplication des ligneux forestiers, fruitiers et ornementaux. Antony, France, Cemagref, p. 183-191.

NOUAIM R., 2005. L'arganier au Maroc : entre mythes et réalités. Une civilisation née d'un arbre. Paris, France, L'Harmattan, 229 p.

PLATTEBORZE A., 1977. Le bouturage des arbres forestiers au Maroc. Bilan des essais réalisés en 1975 et 1976. Annales de la recherche forestière au Maroc, 17 : 145-190.

SAYA R. A., MANKESSI F., TOTO M., MARIEN J. N., MONTEUUIS O., 2008. Advances in mass clonal propagation of Eucalyptus urophylla $\times$ E. grandis in Congo. Bois et forêts des tropiques, $297: 15-25$.

TEKLEHAIMANOT Z., MWANG'INGO P. L., MUGASHA A. G., RUFFO C. K., 2004. Influence of the origin of stem cutting, season of collection and auxin application on the vegetative propagation of African Sandalwood (Osyris lanceolata) in Tanzania. Southern African Forestry Journal, 201 : 13-24. 